\title{
Estimation of the height and angles of orientation of the upper leaves in the maize canopy using stereovision
}

\author{
$\mathrm{N}_{\text {Ivanov }}{ }^{1,2}, \mathrm{P}$ Boissard ${ }^{1 *}, \mathrm{M}$ Chapron $^{2}, \mathrm{P}$ Valéry $^{1}$ \\ 'INRA, Station de Bioclimatologie, F78850 Thiverval-Grignon; \\ ${ }^{2}$ ENSEA, Équipe de Traitement d'Images et du Signal, 1, allée des Chênes-Pourpres, F95014 Cergy-Pontoise, France
}

(Received 24 May 1993; accepted 12 January 1994)

\begin{abstract}
Summary - 3D models of vegetation have been constructed to allow various simulations, such as the interception of incoming radiation, radiative transfer, reflectance simulation in remote sensing, study of plant function, and interspecies competition. The construction of such 3-D models is based upon simple geometric assumptions and several production rules (eg, growing, branching). It poses 2 problems: the acquisition of a large amount of data on leaf heights and orientation angles in the field; and the validation of the model and assessment of its suitability on the scale of the field. This paper presents a method of 3D acquisition and reconstruction based upon stereovision, ie upon the acquisition of data using 2 synchronized CCD or photographic cameras. We first show that Toscani's method of camera calibration can be used under real conditions in the field. We then apply the method to a tall maize canopy $(2.50 \mathrm{~m} \mathrm{high})$ because this canopy allows easy control measurements to be made in the field. Results on the estimation of position and orientation of the leaves are presented. This method allows the estimation of the $X, Y$ and $Z$ coordinates of characteristic points of laminae and stems. The ability of the method to provide inclination angles of the leaves was evaluated to be rather good. Only the leaves belonging to the 4 upper layers were reconstructed because of the difficulties due to hidden areas. This problem is discussed and we show how auxilliary information on crop structure may be complementary to data obtained from stereovision.
\end{abstract}

Zea mays $\mathrm{L}=$ maize / stereovision / 3D structure / geometry of vegetation / leaf height / leaf angle / computer model

\begin{abstract}
Résumé - Estimation de la hauteur et des angles d'orientation des feuilles supérieures d'une culture de maïs à partir de la stéréovision. La mise au point de maquettes 3-D des cultures par voies informatique ou physique répond au souci d'effectuer diverses simulations : interception du rayonnement, échainges radiatifs, simulation de la réflectance en télédétection, étude du fonctionnement de la plante et de la compétition entre espèces. La construction de telles maquettes, fondée sur des hypothèses géométriques simples et sur des règles de production (croissance, ramification,...), pose 2 problèmes: la nécessité d'effectuer de nombreuses mesures au champ (hauteur et orientation des feuilles) et la validation de la maquette à l'échelle de la parcelle. Le présent article décrit une méthode de saisie et de reconstruction 3-D basée sur la stéréovision, c'est-à-dire sur l'acquisition de données à partir de 2 caméras ou de 2 appareils photographiques synchronisés. Dans un premier temps, on montre que la méthode de calibration de Toscani (Toscani, 1988) peut être utilisée en conditions réelles au champ. La méthode est ensuite appliquée à un couvert de maïs adulte (hauteur $2,50 \mathrm{~m}$ ) en raison de la commodité qu'offre ce couvert pour effectuer les mesures de contrôle au champ. On montre que la méthode est pertinente pour l'estimation des coordonnées $X, Y, Z$ de points caractéristiques des limbes et des tiges. La capacité de la méthode à fournir les angles d'inclinaison des feuilles a également été testée et a été jugée acceptable. Seules les feuilles appartenant aux 4 strates supérieures ont été reconstruites en raison du problème posé par les parties cachées. Ce
\end{abstract}

\footnotetext{
${ }^{*}$ Correspondence and reprints

Email : boissard@bcgn.grignon.inra.fr
} 
problème est discuté et on montre en particulier comment des informations extérieures sur la structure de la culture peuvent venir compléter les données issues de la stéréovision.

Zea mays $L=$ maïs / stéréovision / structure 3-D / géométrie de la végétation / hauteur des feuilles / angle des feuilles / maquette informatique

\section{INTRODUCTION}

The description of the geometric structure of vegetation canopies and, particularly, crops has several applications at present. Firstly, crop-simulating models are intended for agrometeorological purposes on a large scale (Whisler et al, 1986; Brisson et al, 1992) or for testing different agronomical conditions on a smaller scale (Porter, 1984). Both require structural parameters describing variables at given stages, such as leaf area index (LAI) or percentage of soil cover. Secondly, radiative transfer simulation models inside the canopy require structural parameters such as LAI per horizontal layer and leaf inclination angle distribution. This type of model, eg, the SAlL model (Verhoef, 1984), is particularly important in remote sensing since model inversion is a means of retrieving the relationship: spectral reflectance $=f$ (target characteristics).

In the models described above the plant geometry is rather poor since uniform variables such as LAl are used. However, computer vegetation models, which give a good representation of 3D geometry (de Reffye et al, 1988), are necessary to study the interception of incoming solar radiation, directional reflectance simulation, interspecies competition phenomena, and disease propagation. This type of model is based on a geometric and topological architecture modeling of plant populations and requires a large number of in situ measurements, which may be time-consuming. Computer vegetation $3 \mathrm{D}$ models of maize, constructed from mathematical rules or statistical distributions, have been proposed (Goel et al, 1991; Prevot et al, 1991). These are also based on numerous measurements of lengths and inclination angles in the field.

Various methods have been proposed to assess global structural parameters that do not explicitly refer to individual plants or leaves. For instance, the following parameters can be estimated: the LAI using light penetration inside the canopy (Welles and
Norman, 1991); the percentage of soil cover using radiometric data (Boissard et al, 1992); and the directional gap fraction using hemispherical photos (Bonhomme and Chartier, 1972) or a laser distance meter (Sinoquet et al, 1991, 1993).

However, various attempts have also been made to measure parameters related to the fine 3D structure, such as the shape and the relative position of phytoelements : $2 \mathrm{D}$ plant profiles (Bonhomme and Varlet-Grancher, 1978); 3D digitizer (Moulia and Sinoquet, 1993); manual photogrammetric measurements (Boissard, 1985; Lewis and Muller, 1990); and phytoelements locating using radiance measurements (Andrieu and Ivanov, 1993).

New techniques for 3D reconstruction of plants using stereovision have been proposed (Jia and Krutz, 1992). This technique was applied to artificial plants $25 \mathrm{~cm}$ high (Chapron et al, 1992b). The resulting 3D computer model is then used for ray-tracing [simulation of the path of the light ray into the canopy] analyses (Chapron et al, 1993).

Some of the existing methods are destructive (plant profile method) or time-consuming (3D digitizer). Others are useless for some kinds of canopy because of the limitations for man movement inside the canopy (maize) or the difficulty of carrying out measurements. For instance, the use of a clinometer to determine the angle between the lamina plane and the vertical direction is quite difficult. The major reason for developing a stereovision approach was thus to bypass the drawbacks of the existing methods for characterizing plant structure.

Automatic procedures applied to stereodata acquired from airborne or satellite platforms are now used on an operational basis (de Masson d'Autume, 1984; Cappellini et al, 1991). Unfortunately, this scale is quite far from a close-range description of vegetation. Liu (Liu, 1991; Chapron et al, 1992a) proposed a method for 3D automatized object reconstruction in a polyhedric environment (fig 1). He analyzed objects such as rather simple and regular volumes with planar sides 


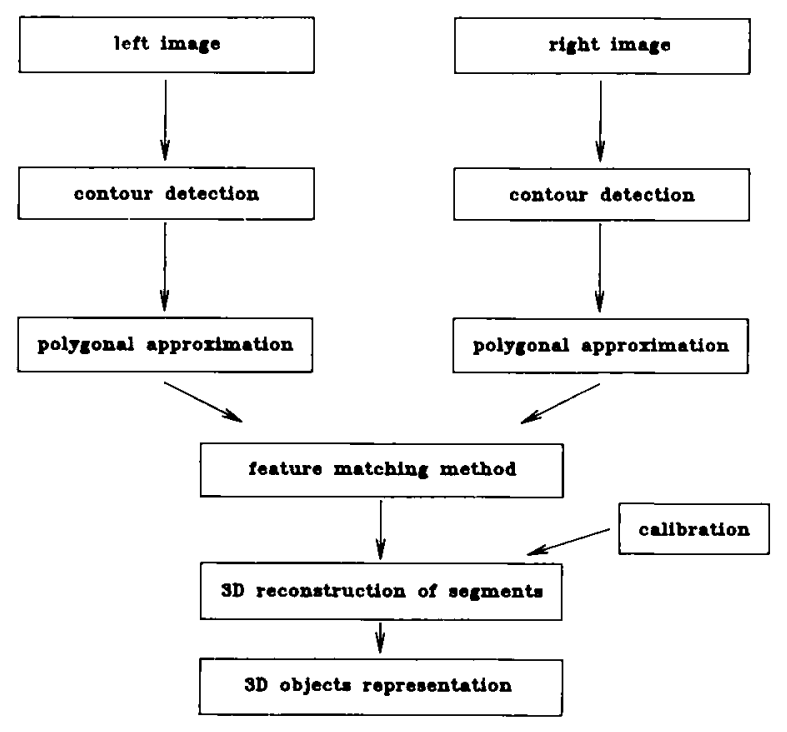

Fig 1. The steps of automatic matching in stereovision and $3 \mathrm{D}$ reconstruction of objects in a polyhedric environment (after Liu, 1991).

limited by straight edges. In contrast to this situation, computer vision applied to plants must take into account discontinuities due to thin objects of limited size, distributed in the 3D space. For instance, considering a transect on one image of the canopy, discontinuities of the $X, Y$, or $Z$ coordinates of the phytoelements are encountered on the edges of the leaf laminae. Computer vision applied to such a medium must take into account the following aspects: leaves hide each other from the camera; and when 2 cameras are used, leaves or parts of the leaves that are seen by one camera may be not seen by the other.

As a consequence, the nondestructive description of the canopy geometry based on stereovision is limited to the upper layers of the canopy. Such a description is useful for light interception estimates or evapo-transpiration studies since the upper layers are of a great importance in the mechanisms involved. However, this description is unlikely to be sufficient to account for vegetation canopies in which stress is laid on the lower leaf layers (vegetation under trees in forest, or near infrared light penetration).

A first attempt to apply Liu's procedure to automatized plant reconstruction was performed by Ivanov (1991) and did not succeed because of problems arising from the contours of leaves and the left-right matching of the images in a discontinuous medium. The approximation of the leaf contours by line segments thus appeared to be unsuitable because the contour shape is rather convex with a regular curvature. The present paper describes a new manual approach to plant identification, image matching and final 3D reconstruction based on stereovision. This allows the following steps to be carried out: a feasibility study of stereovision using data handling by an operator to carry out digitization of the leaves and matching; the set-up of acquisition parameters; and the estimation of variables describing the basic structure such as leaf height and orientation angle.

\section{MATERIALS AND METHODS}

Papers dealing with stereovision generally present results obtained indoors with $2-3$ video $C C D$ cameras (Faugeras, 1988; Chapron et al, 1992b). For our purposes, classical CCD cameras $(512 \times 512$ pixels) proved to have low resolution. A technique based on stereophotography was thus chosen and combined with 2D digitization of paper enlargements.

\section{Data acquisition}

Two Hasselblad electric cameras, format $6 \times 6 \mathrm{~cm}$, linked to a synchronization device, were fixed to a horizontal bar, at a height $H$ of $7.80 \mathrm{~m}$ above the maize canopy. The camera lenses were $2 \mathrm{Carl}$ Zeiss Planar $3.5 \mathrm{~s}, f=10 \mathrm{~cm}$, with a plane aperture angle of $30^{\circ}$. A base length $B$ of $0.75 \mathrm{~m}$ between the cameras was chosen. The 2 optical axes were vertical and the overlap area was $4 \times 3.25 \mathrm{~m}$ measured at ground level. A slide colour film (Kodak Ektachrome 200 ASA) was used. Enlargements on paper (fig 2) were performed on 2 scales $(18 \times 18 \mathrm{~cm}$ and $40 \times 40 \mathrm{~cm})$.

\section{Plant material}

The vegetation canopy was Zea mays $L$, DEA cultivar, at normal density $(0.8 \mathrm{~m}$ between rows and $0.15 \mathrm{~m}$ along rows). Photographs and measurements were made after anthesis when the crop had reached its maximum height $(250 \mathrm{~cm})$. Maize plants had about 11 leaf layers at anthesis and, of these, about 9 were still green at the date of the experiment. A large number of leaves in the observed volume were identified with marks directly painted on the upper face with white glycero paint (fig 2). The height and the inclination angles of laminae of leaves were measured at each mark. A digital clinometer was used to obtain the inclination angle of the normal to the leaf plane. 


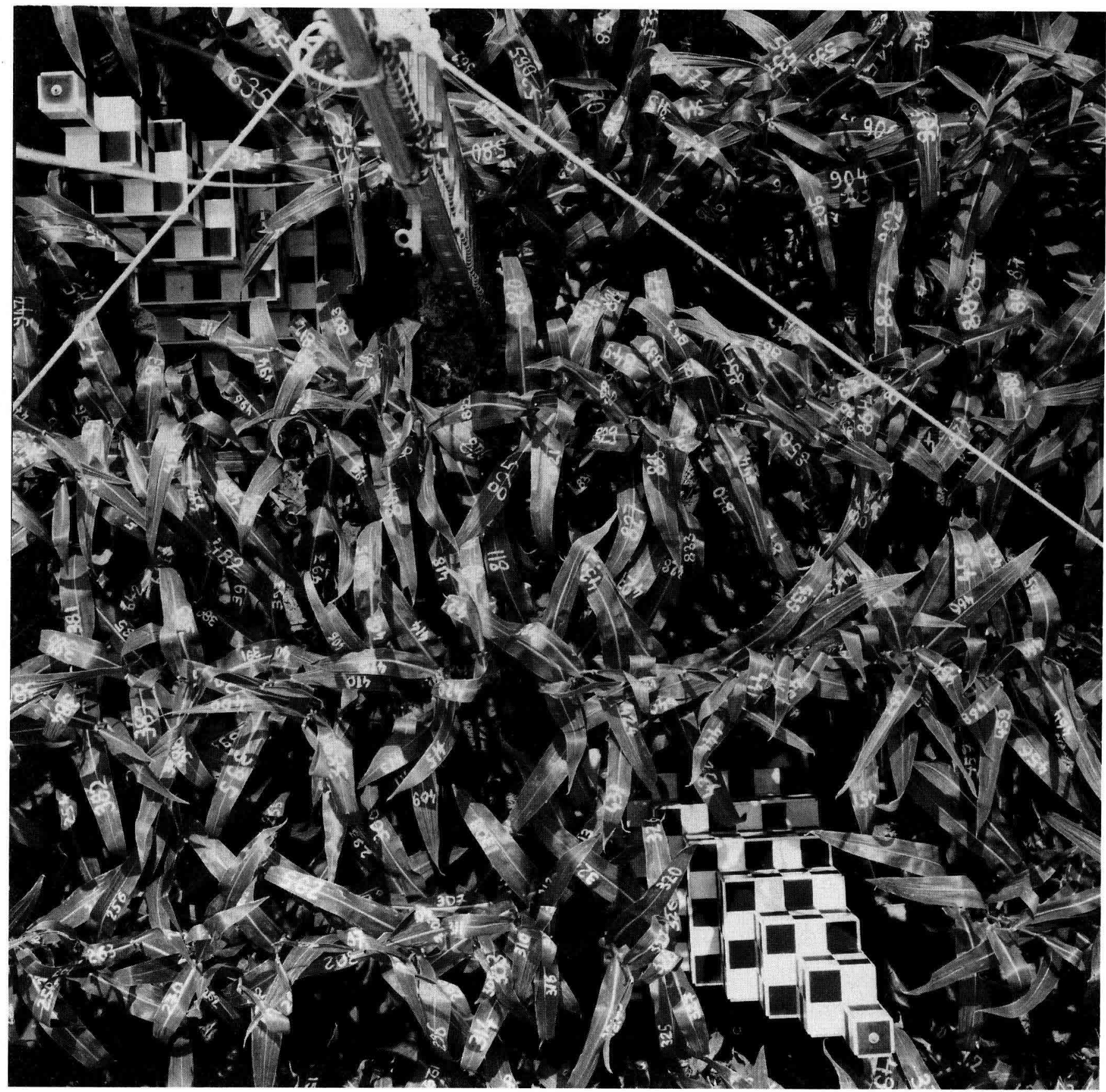

Fig 2. Vertical photograph of a maize canopy after anthesis taken at a height of $8 \mathrm{~m}$ showing identified leaves (painted marks) and 2 reference targets.

\section{Description of the $3 D$ stereovision method}

\section{Principle}

Figure 3 presents the principle of the stereovision (Faugeras, 1988). If the position of the 2 cameras in the $3 D$ space is known, then it is possible to determine the $X, Y, Z$ coordinates of a given point $M$ by calculating the intersection of the 2 homologous rays $m_{1} M, m_{2} M$. The nadir points $n_{1}$ and $\mathrm{n}_{2}$ represent the intersection of the optical axes with the image plane. The difference $m_{1} n_{1}-m_{2} n_{2}$ is called the linear disparity or disparity, while the parallax $P$ of $M$ is defined by $P=m_{1} m_{2}$. Each camera position and homologous ray equation is calculated using a set of parameters specific for each camera. These parameters are retrieved during the calibration procedure described below. 


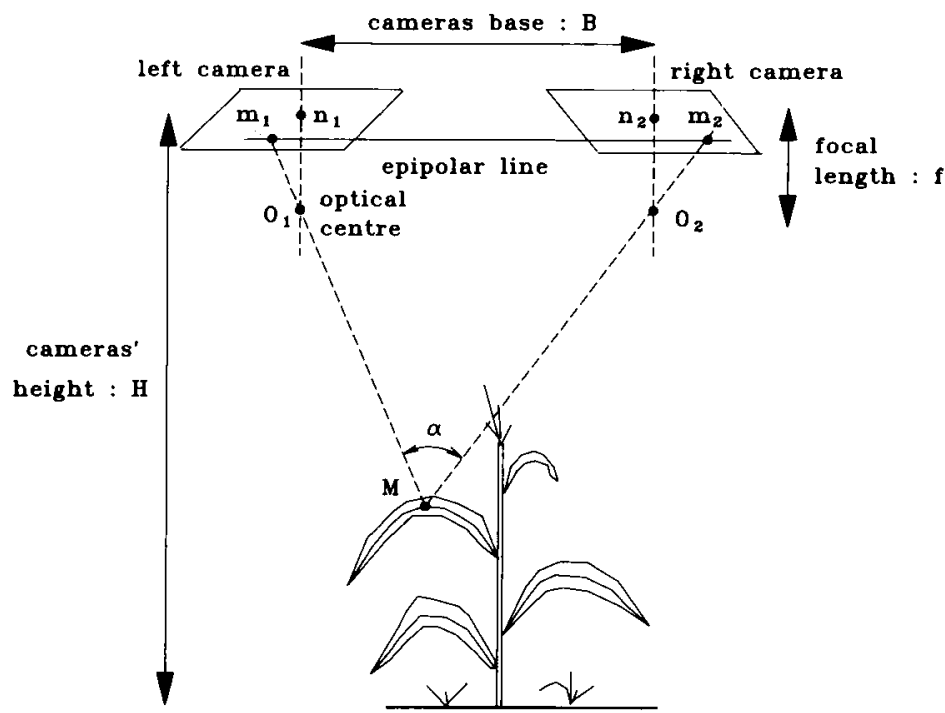

Fig 3. General principle of stereovision in order to assess the position of phytoelements in 3D space.

\section{Calibration of system}

The calibration method proposed by Toscani (1988) assumes that each camera may be modelized according to a pin-hole model (fig 4), ie the camera performs a perfect perspective transform, whose centre is the lens optical centre. The calibration consists of calculating the elements of the 3 lines $x 4$ columns matrix $A$, the 'perspective transform matrix'. A describes the relationship between the camera and the scene and is required for the 3D reconstruction of the objects studied. Its elements are computed by using a set of calibration points which are also called reference points. There are 3 different reference systems to be considered in stereo computing (fig 4):

- $(O, u, v)$ associated with the image plane;

- $\left(O_{1}, X_{1}, Y_{1}, Z_{1}\right)$ associated with camera No 1;

- $\left(O_{w}, X_{w}, Y_{W}, Z_{w}\right)$ associated with the 3D scene.

A calibration point $P$ is considered in figure 4 . The goal is to find the relationships between its $3 \mathrm{D}$ posi-

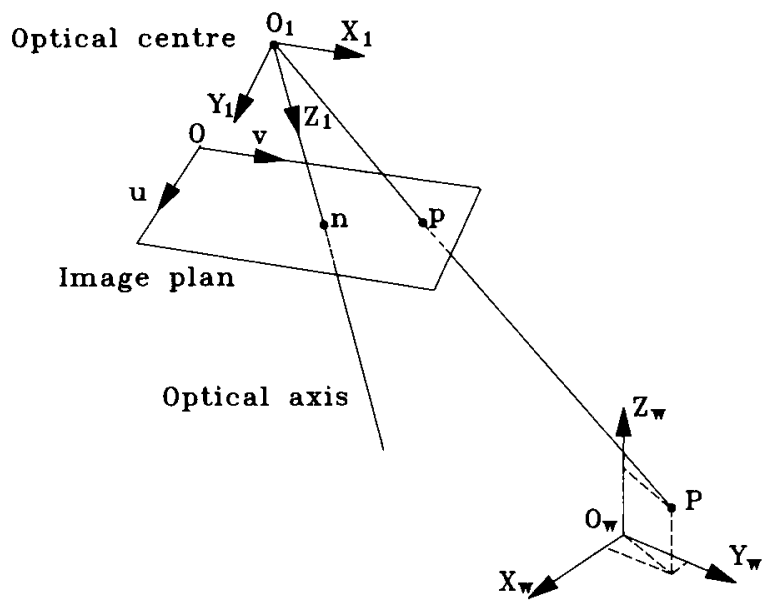

Fig 4. Display of the 3 systems of coordinates referencing the 3D space: ( $\left.O_{W}, X_{W}, Y_{W}, Z_{W}\right)$ linked to the 3D scene; $\left(O_{1}, X_{1}, Y_{1}, Z_{1}\right)$ linked to the camera No $1 ;(O, u, v)$ linked to the image plane. tion $\left(X_{W P}, Y_{W P}, Z_{W P}\right)$ and the $\left(u_{p}, v_{p}\right)$ coordinates of its corresponding point $p$ in the recorded image. Toscani (1988) proposed the following equation:

$$
\left(\begin{array}{c}
u_{p} \\
v_{p} \\
1
\end{array}\right)=\mathbf{A}\left(\begin{array}{c}
X_{W P} \\
Y_{W P} \\
Z_{W P} \\
1
\end{array}\right)
$$

where $\mathbf{A}$ is the perspective transform matrix. $\mathbf{A}$ is calculated by applying equation [1] to several reference points during the calibration procedure. There are 2 steps for each reference point. The first step is to find the relationship between the systems $\left(O_{1}, X_{1}, Y_{1}, Z_{1}\right)$ and $\left(O_{W}, X_{W}\right.$, $\left.Y_{W}, Z w\right)$; the position of $P$ in the camera system is related to its position in the scene by using a rotation, followed by a translation:

$$
\left(\begin{array}{c}
X_{1 P} \\
Y_{1 P} \\
Z_{1 P} \\
1
\end{array}\right)=\mathbf{R}\left(\begin{array}{c}
X_{W P} \\
Y_{W P} \\
Z_{W P} \\
1
\end{array}\right)+\mathbf{T}
$$

where $\mathbf{R}$ and $\mathbf{T}$ are the rotation and translation matrix, respectively.

The second step is to find the relationship between the systems $\left(O_{1}, X_{1}, Y_{1}, Z_{1}\right)$ and $(O, u, v)$; the coordinates of $P$ in the $3 D$ system linked to the camera No1 are $\left(X_{1 p}, Y_{1 p}, Z_{1 p}\right)$. The basic geometric relationships between the coordinates of $\mathrm{P}$ in the camera's reference system and $u_{p}, v_{p}$ are:

$$
\frac{f}{Z_{1 P}}=\frac{u_{p}}{X_{1 P}}=\frac{v_{p}}{Y_{1 P}}
$$

The 12 elements of the $\mathbf{A}$ matrix are calculated by using $N$ reference points $(N>6)$. The application of [1] to the coordinates of these points $\left(X_{W i}, Y_{W i}, Z_{W i}\right.$, in the 3D space and $u_{i}, v_{i}$ in the image, $i=1 \ldots N ; w=1,2$ ) results in a $2 N$-equation linear system. A is then calculated by using Greville's (1960) inversion method. The elements of the A matrix are related to 10 independent par- 
ameters containing information about the cameras and the scene. Of these parameters, 4 are intrinsic, ie linked to the camera (coordinates of the image centre and focal length), and 6 are extrinsic, ie relating the camera to the $3 D$ scene (translation and rotation).

The $N$ reference points are obtained from 2 calibration targets, $1.8 \mathrm{~m}$ high, which are placed on the ground inside the vegetation canopy (fig 2). Each target consists of 6 wooden parallelepipeds (each $0.3 \mathrm{~m}$ high) having increasing horizontal surface so that they can be placed vertically one above the other. A black-and-white check pattern was painted on 2 sides of each parallelepiped, which allows the identification of 162 squares on the photo of each target. The positions of the corners of each white square are known in the 3D space and are used as reference coordinates.

\section{Digitization of leaf marks on stereo-pairs}

About 100 leaves with their identification number visible on both right and left photographs were used in order to validate the method. Working on enlargements, an operator digitized 4-5 points per leaf and per photo; reference points were chosen on painted lines (marks) at the place of changing direction or extremities of marks. To do this, enlargements were fixed to the digitizer so as to have the $X$ axis corresponding to the epipolar direction (fig 3 ) and the $Y$ axis perpendicular to it. Pairs of points belonging to the left and right images were determined in order to be reconstructed in the 3D space. Two scales were tested for enlargements (coefficients: $\times 3$ and $\times 6.5$ ). However no significant difference was observed in the accuracy of results.

\section{Reconstruction of phytoelements in 3D space}

This method can be used to reconstruct the phyto elements viewed by the 2 cameras in the 3D space. Homologous contours of leaves are identified and digitized on both photos. In order to match the candidate points belonging to homologous contours (leaf edge), 2 rules are used: (i) the candidate points are located on the homologous edges of a given leaf (right or left edge); and (ii) the candidate points are both situated on an epipolar straight line, the direction of which is known (same direction as $n_{1} n_{2}$ nadir line) because the optical axes are parallel (fig 3 ).

\section{RESULTS}

The different sets of points used for calibration purposes and for testing the accuracy of the method are presented in table I.

\section{Perspective transform matrices}

The perspective transform matrix $\mathbf{A}$, associated with the camera, was calculated separately for each camera, which consists of evaluating 12 elements. These elements are associated with 10 parameters (table II). The values of some parameters or relationships between them were known: focal length, height of cameras, base length and image size. This allows the control of the accuracy of the calibration. For example, the estimated values of the cameras height were $7.65 \mathrm{~m}$ and $7.69 \mathrm{~m}$ versus $7.8 \mathrm{~m}$ measured, which is rather good. As far as focal length is concerned, we found about $95 \mathrm{~mm}$ versus a theoretical value of $100 \mathrm{~mm}$.

Table I. Number of point pairs used for calibration purposes and for testing the accuracy of the method.

\begin{tabular}{lcc} 
Purpose & $\begin{array}{c}\text { Reference } \\
\text { points } \\
\text { on target }\end{array}$ & $\begin{array}{c}\text { Reference } \\
\text { points } \\
\text { on canopy } \\
\text { (leaf marks) }\end{array}$ \\
\hline Calibration & 40 & \\
Test of accuracy & 40 & 460 \\
Height & & 25 \\
Zenith & & 100 \\
Azimuth & & \\
\hline
\end{tabular}

Zenith and azimuth angles define the direction of the normal to the leaf surface

Table II. Values of intrinsic and extrinsic parameters defined by the perspective transform matrix.

\begin{tabular}{lcc}
\hline & Left image & Right image \\
\hline Intrinsic parameters & & \\
Image centre (cm) & & \\
$u$ axis & 10.1 & 9.9 \\
$v$ axis & 8.7 & 8.9 \\
Focal length (mm) & & \\
$u$ axis & 103.0 & 103.1 \\
$v$ axis & 102.5 & 102.6 \\
Extrinsic parameters & & \\
Translation (cm) & & \\
$x$ axis & -83.6 & -157.7 \\
$y$ axis & 143.1 & 131.4 \\
$z$ axis & 765.2 & 768.5 \\
Rotation $\left({ }^{\circ}\right)$ & -1.6 & -2.7 \\
$x$ axis & 0.50 & 0.14 \\
$y$ axis & -0.6 & -0.5 \\
$z$ axis & & \\
* Calculated for enlargements on paper & $(18 \times 18 \mathrm{~cm}$, \\
factor $x 3)$ &
\end{tabular}




\section{Target reconstruction}

In order to validate the method, 40 points belonging to targets were reconstructed by the model and compared with their real positions. No bias was observed on $X, Y, Z$ coordinates and the standard deviations were as follows:

$\sigma(X)=\sigma(Y)=1 \mathrm{~cm}$ for $X$ (and $Y)(0.4 \%$ of target length on the $X$ (or $Y$ ) axis);

$\sigma(Z)=6 \mathrm{~cm}$ for $Z(3.0 \%$ of target height on the $Z$ axis).

The equations of regression constrained to the origin are:

$X_{\text {estimated }}=1.00 X_{\text {measured }}\left(r^{2}=1.00, n=108\right)$

(same for $Y$ )

$Z_{\text {estimated }}=1.03 Z_{\text {measured }} \quad\left(r^{2}=0.99, n=108\right)$

\section{Estimation of leaf height at mark location}

Painted marks on leaves were used to test the ability of the method to assess the heights of phytoelements. A set of 460 points belonging to 98 leaves was reconstructed and their $Z$ coordinates compared with their real heights (fig 5). The standard deviation $\sigma(Z)$ rises to $12 \mathrm{~cm}(4.8 \%$ of canopy height). This is not surprising since leaves are not rigid and the reference height zero was inaccurate because the ground did not have the regularity of a horizontal plane. The equation of regression constrained to the origin is:

$Z_{\text {estimated }}=0.98 Z_{\text {measured }} \quad\left(r^{2}=0.96, n=460\right)$

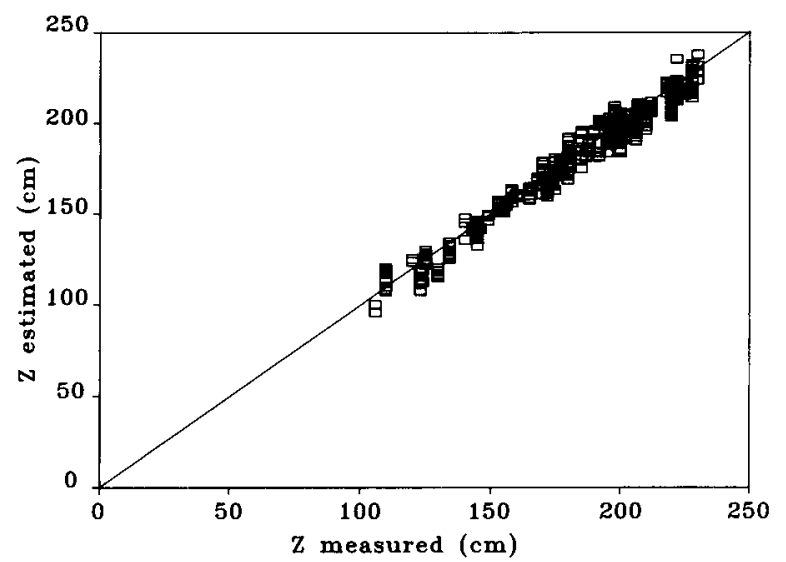

Fig 5. Practical evaluation of accuracy of the reconstruction of maize leaves: scatter plot of $Z$ estimates of 460 points belonging to 100 leaves.

\section{Orientation angles of leaves}

Two kinds of orientation angles were studied: the zenith inclination angle $\theta$ and the azimuth angle $\varphi$.

The orientation angles of the laminae planes of leaves at the location of the marks were estimated from stereovision and compared to in situ measurements. We tested the ability of the method to retrieve the inclination angle $\theta$ of the normal to the surface at a given location on the leaves and the azimuth angle $\varphi$ of the leaf main veins. Inclination angles $\theta$, ie the angle between the normal to the leaf and the vertical direction, were calculated for 25 leaves. For each leaf a tangent plane defined by $5-6$ points belonging to the leaf marks was constructed. The normal direction to this plane then gave the inclination angle $\theta$ of this part of the leaf. Figure 6 shows the result of estimates against the real values as measured by the clinometer. The standard deviation $\sigma(\theta)$ was $7^{\circ}$. The equation of regression constrained to the origin was:

$\theta_{\text {estimated }}=0.98 \quad \theta_{\text {measured }} \quad\left(r^{2}=0.79, n=25\right)$

As regards the azimuth angle $\varphi$, we tried to retrieve the direction of the vertical plane including the main vein of the leaf at given locations on the leaves (marks). The average direction of both edges and the main vein were directly estimated from one photo by simply using a protractor. The azimuth of the projection of the main vein on the horizontal plane was calculated using the 3D model from several reconstructed points. The com-

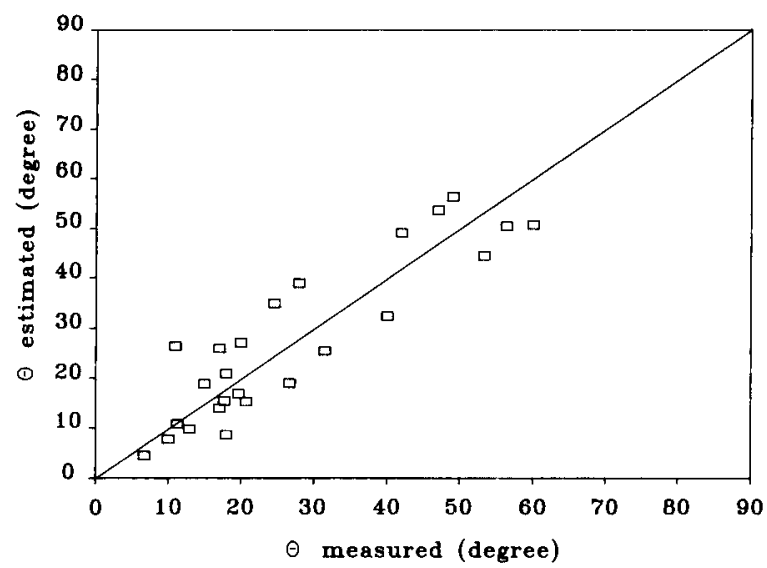

Fig 6. Practical evaluation of accuracy of the reconstruction of maize leaves: scatter plot of angles of inclination of leaves estimates. 
parison is only exact when the main vein and the normal to the lamina both belong to the same vertical plane. Figure 7 gives the comparison between estimates using the $3 D$ model and protractor measurements on 100 points. We calculated a standard deviation $\sigma(\varphi)$ of $2.5^{\circ}$. The equation of regression constrained to the origin was:

$$
\varphi_{\text {estimated }}=1.00 \varphi_{\text {measured }} \quad\left(r^{2}=0.99, n=130\right)
$$

This result is in good agreement with previous results, showing that the estimates using $X, Y$ coordinates (horizontal plane) are better than those using $Z$.

\section{Reconstruction in 3 dimensions}

The stereovision method was used in the field to reconstruct the leaves of the upper layers of maize plants. Only the 4 upper leaf layers were reconstructed in the 3D space (fig 8). To do this, all edges of the leaves (or part of them) were digitized on both photographs. The resulting files contain pairs of contours corresponding to individual leaves which have been recognized and extracted from the 2 images.

However, the data must be corrected in order to eliminate false contours generated in the image plane by the superimposition of leaves at different $Z$ altitudes. A contour is a common limit between 2 objects represented by their projections in the image plane. In the 3D space, the upper leaf may partially hide its neighbour below, generating false contours for the lower leaf which are not physically true and must be removed. To do this

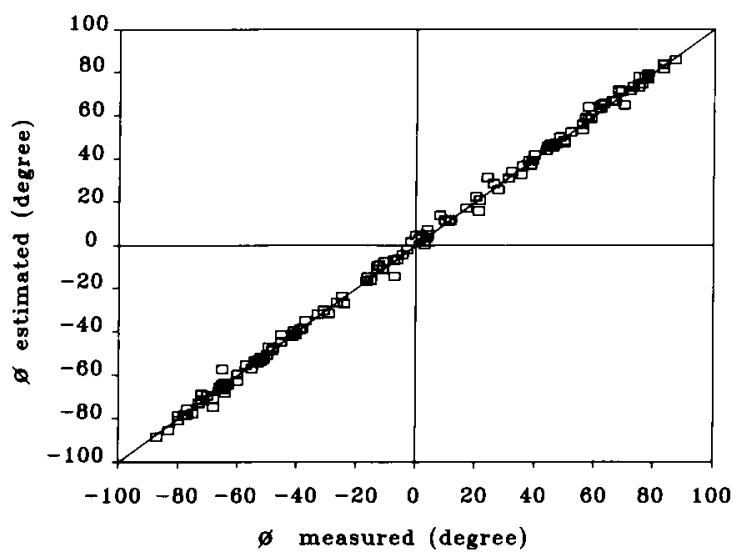

Fig 7. Practical evaluation of accuracy of the reconstruction of maize leaves: scatter plot of azimuth angles of leaf estimates. we developed an automatic algorithm based on the hierarchy along the $Z$ axis, which is established from measurements of disparities $m_{1} m_{2}$ along epipolar lines (fig 3 ). Conversely local breaks of a contour line due to occlusion by another object must be restored, using an interpolation algorithm.

It should be noted that the stems have been reconstructed, although they are not visible on the original photographs. In fact they have been restored from another pair of photographs, taken after all the leaves had been picked off manually. In figure 8 individual leaves are represented by a collection of adjacent triangles so that the surface of the leaves can be calculated.

\section{DISCUSSION AND CONCLUSIONS}

\section{The method}

In order to discuss the relevance of the proposed stereovision method to 3D geometry analysis of maize, we compared the accuracy of leaf-layer reconstruction using the average distance between 2 consecutive layers of real plants. To do this we determined an average plant profile: the distance between 2 consecutive alternate leaves is about $20 \mathrm{~cm}$ and there is $40 \mathrm{~cm}$ between 2 non-alternate leaves on the same side. The confidence interval on height $( \pm 12 \mathrm{~cm})$ from stereovision

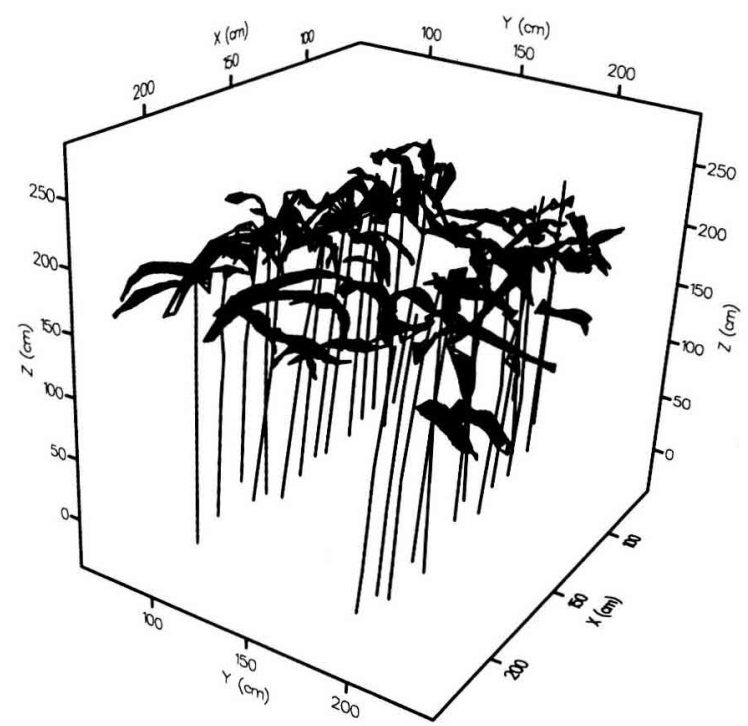

Fig 8. Tridimensional reconstruction of the 4 upper layers and the stems of a maize canopy using stereovision data. 
should thus be compared to 20 or $40 \mathrm{~cm}$ between 2 leaves. This accuracy seems to be good enough to allow the unambiguous separation of 2 consecutive levels. On the other hand, our experiment indicated that the accuracy of the $Z$ estimates is more crucial than the accuracy of $X$ or $Y$. The distortion and the noise affecting the 3D data are due to 3 causes: distortion brought about by camera and enlarger lenses; distortion due to photographic processing; and noise from manual digitization ( $\pm 0.2 \mathrm{~mm}$ ).

Distortion due to lenses appeared to be quite low. Two solutions have thus been considered in order to compensate for the remaining causes of noise: the smoothing of the edges of reconstructed leaves in the 3D space; and the optimization of the system parameters.

\section{Improvement of accuracy by optimization of system parameters}

The accuracy of $X, Y, Z$, and therefore $\theta, \varphi$ estimates depends on 3 main parameters: the focal length $f$ of the cameras, the distance $B$ between the cameras and their height $H$. It also depends on the noise $\delta u, \delta v$ affecting the image coordinates $u$ and $v$. Since $f$ is a lens characteristic and $\delta u, \delta v$ depend on the digitization table and the skill of the operator, we first simulated the resulting noise produced by the error of $\delta u, \delta v$ for different values of $B$ and $H$ parameters on the $Z$ coordinate. Gaussian noise on $u, v$ image coordinates of 100 points chosen in the vegetation volume was simulated practically (with $\delta u=\delta v \approx 0.2 \mathrm{~mm}$ ); these points were then reconstructed. Figure 9 shows a plot of the value of the standard deviation $\sigma(Z)$ of the $Z$ coordinate against the camera base $B$ for different values of camera height $H$. The trend of the curve shows that $\sigma(Z)$ strongly increases for $B<0.60 \mathrm{~m}$ and $H=8 \mathrm{~m}$. This result allowed us to use the following parameter values for the experiment: $B=0.75 \mathrm{~m}$ and $H=8 \mathrm{~m}$. In these conditions, the angle $\alpha$ of 2 homologous rays meeting at the centre of the common area (fig 3 ) varies from 5 to $8^{\circ}$ according to leaf height. This angular configuration means that the 2 cameras acquire 2 slightly different images. These differences were quantified: $5 \%$ of leaf edges are lost because they are detected by only one camera.
Let us note that the fact that homologous rays are almost vertical and meet at an acute angle mainly explains the difference observed between $X$ and $Y$ accuracy and $Z$ accuracy.

Another method is the analytical approach. Let us write the analytical relationship between the parallax $P$, the height $H$ and the system parameters $B$ and $f$, as proposed by Caillemer (1967). Figure 10 presents the construction in the image plane of the points $M$ and $\mathrm{N}$ located on the same vertical line; $\mathrm{O}_{1}$ and $\mathrm{O}_{2}$ are the optical centres. Since the projections $m_{1}$ and $n_{1}$ are superposed, the linear parallax is given by:

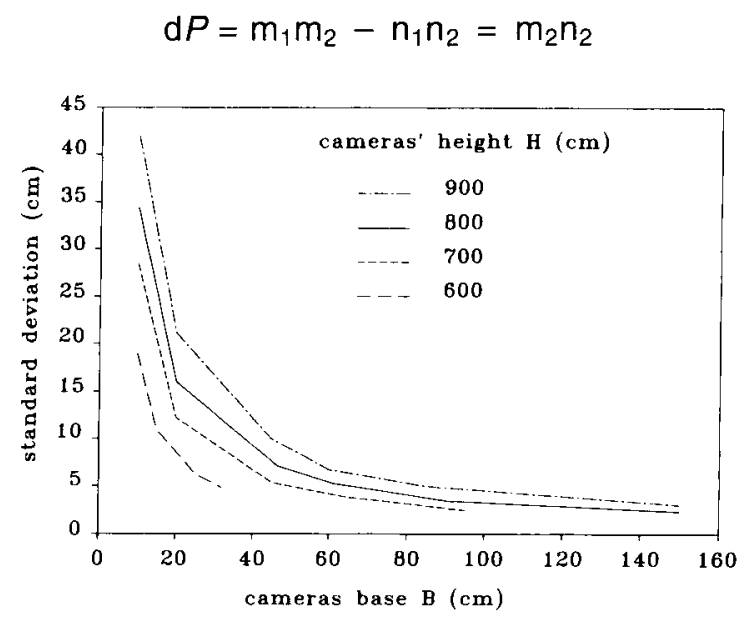

Fig 9. Sensitivity study of the 3D reconstruction model as a function of the cameras extrinsic parameters (height and base). The standard deviation measures the dispersion of 100 reconstructed points, randomly spread in the field.

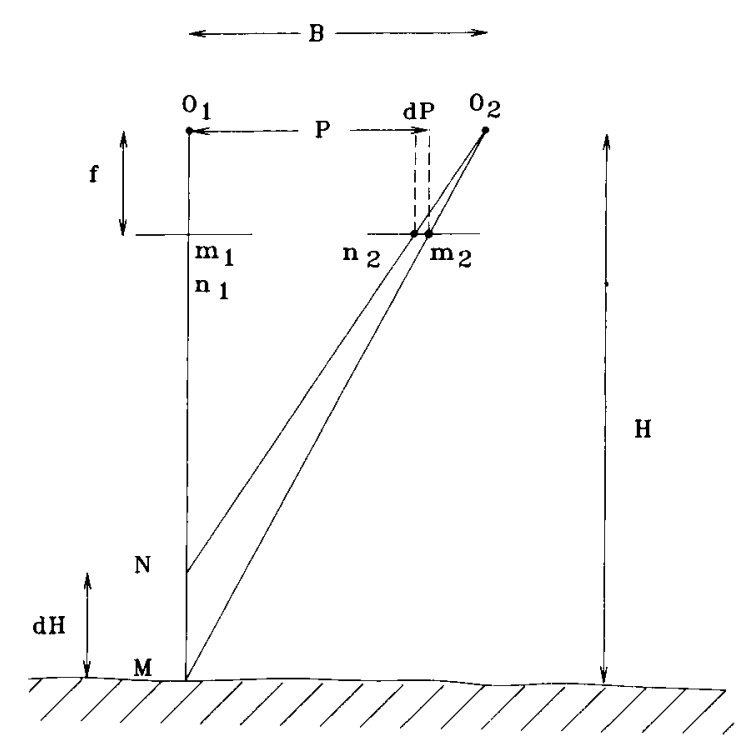

Fig 10. Construction in the image plane of 2 points $M$ and $\mathrm{N}$ located on the same vertical line and measurement of their respective parallax $m_{1} m_{2}$ and $n_{1} n_{2}$. 
We get:

$$
\frac{P}{B}=\frac{H-f}{H}=1-\frac{f}{H}
$$

and:

$$
P=B-\frac{B f}{H}
$$

For a point $\mathrm{N}$ located at $\mathrm{d} H$ from the altitude of $\mathrm{M}$, we have a parallax $\pm \mathrm{d} P$. Differentiating the expression of $P$, we obtain:

$$
\mathrm{d} P=-\frac{B f}{H^{2}} \mathrm{~d} H
$$

and

$$
\mathrm{d} H=-\frac{H^{2}}{B f} \mathrm{~d} P
$$

Numerical values of the parameters under our conditions are given in table III. For the error $\mathrm{d} H$ (maximum error estimated at the ground level) we obtain:

$$
\mathrm{d} H=5.4 \mathrm{~cm}
$$

This value is in good accordance with the standard deviation of the $Z$ coordinate $\sigma(Z)=6 \mathrm{~cm}$. Improving the accuracy of $\mathrm{d} H$ means decreasing $H$ or increasing $B$ values. The expected effects will be to reduce the extension of the viewed area or to make it more difficult to reconstruct the hidden areas respectively. As regards the reconstruction of the lower layers, directional gap frequency analysis (Andrieu, personal communication) designed for radiative transfer modeling can solve the problem of how many leaves (or leaf parts) are viewed from 2 distinct directions of view.

\section{Reconstruction of hidden elements of the canopy}

Plant geometry cannot be represented as classical polyhedric objects. As a consequence it is necessary to adapt the stereo-

Table III. Experimental values of stereovision system parameters.

\begin{tabular}{ll}
\multicolumn{1}{c}{ Parameter } & Value \\
\hline dp $(\mathrm{mm})$ & \\
enlargement & 0.2 \\
film & 0.067 \\
$H(\mathrm{~m})$ & 7.8 \\
$B(\mathrm{~m})$ & 0.75 \\
$f(\mathrm{~m})$ & 0.1 \\
\hline
\end{tabular}

$\mathrm{dp}$ : parallax increment; $\mathrm{H}$ : height; $\mathrm{B}$ : base; $f$ : focal length. vision approach to plant specificity. First, introducing additional information on plant (phyllotaxis) or population structure seems to be of great interest. In the future, the development of some automatic help in order to make processing more rapid will be necessary so that this new approach can be considered as operational.

Since individual elements must be imaged by 2 cameras, only the upper layers are candidate to a total or partial reconstruction. It was demonstrated that about $5 \%$ of the leaf edges are not viewed simultaneously by the 2 cameras and are lost for the 3D model in the case of $B / H .=1 / 10.7$. Figure 11 presents hidden areas of leaves in the case of a maize canopy reduced to 4 leaves and photographed in the conditions described in Materials and methods. This figure suggests that we have enough information to reconstruct 4 layers, as has been proposed by Chapron et al $(1992 b, 1993)$ in the case of

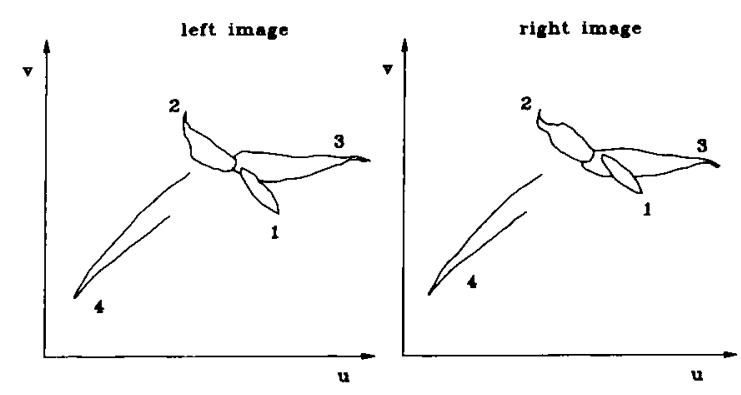

VIEWED COMMON PARTS FROM TWO VERTICAL STERBOSCOPIC CAMERAS

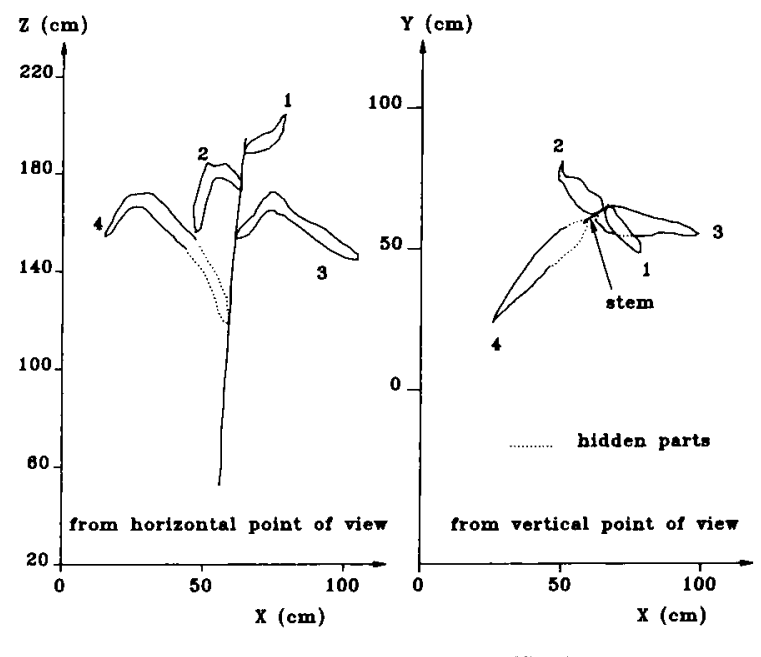

3D RECONSTRUCTION OF FOUR LKAVES

Fig 11. Tridimensional reconstruction of a maize plant combining information on viewed parts with information on insertion coordinates of leaf No 4 . This information is inferred from the row position and the general shape of a maize leaf. 
maize. The insertion of the leaf No 4 may be inferred from the row position and the general shape of a maize leaf (Prevot et al, 1991; Prevot and Brunet, 1993). Since leaves are vertically distributed along the stem, we will not distinguish between the layer concept and the order of leaves along the stem. Thus, for each group of 4 consecutive layers, the reconstruction of hidden areas should be obtained by combining 3-D partially reconstructed leaves with some a priori knowledge of maize structure (for instance, height of insertion points on stem, average shape). Generalizing this statement leads to the capability of reconstructing the whole structure. The first $n$ leaves are manually picked off after being photographed, thus allowing access the $n$ following leaves. Notice that the capability of reconstruction of partly hidden leaves must be tested for each type of structure.

\section{ACKNOWLEDGMENTS}

We would like to give special thanks to the following: A Allix, B Andrieu, R Bonhomme, $\mathrm{P}$ Cellier, $B$ Durand, F Laroque, S Masson, JG Pointel, D Renard, and $Y$ Tymen, who helped us to perform this work.

\section{REFERENCES}

Andrieu B, Ivanov N (1993) Use of radiance measurements to assess the position of phytoelements in a vegetation canopy. Agric For Meteorol 66 139-157

Boissard $P$ (1985) Description de la géométrie d'une culture avec restriction aux parties vues. Proc 3rd Int Colloq Spectral Signatures of Objects in Remote Sensing, Les Arcs, France, 1620 December, ESA SP-247, 209-212

Boissard P, Pointel JG, Tranchefort J (1992) Estimation of the ground cover ratio of a wheat canopy using radiometry. Int $J$ Remote Sens $13,1681-1692$

Bonhomme R, Chartier M (1972) The interpretation and automatic measurement of hemispherical photographs to obtain sunlit foliage area and gap frequency. Isr J Agric Res 22, 53-61

Bonhomme R, Varlet-Grancher C (1978) Estimation of gramineous crop geometry by plant profiles including leaf with variations. Photosynthetica $12,193-196$

Brisson N, King D, Nicoullaud B, Ruget F, Ripoche D, Darthout R (1992) A crop model for land sui- tability evaluation: a case study of the maize crop in France. Eur J Agron 1, 163-175

Caillemer A (1967) Topographie, photogrammétrie. Publications de l'Institut Français du Pétrole, Ed Technip, Paris, $315 \mathrm{p}$

Cappellini V, Alparone L, Galli G, Langé P, Mecocci A, Menichetti L, Capanni G Carlà R (1991) Digital processing of stereo images and 3D reconstruction techniques. Int $J$ Remote Sens, 12, 477-490

Chapron M, Cocquerez JP, Liu N (1992a) Precision of camera calibration and stereovision performed by standard cameras and image digitizer. 11th IAPR ICPR, August 30 - September 3, The Hague (The Netherlands)

Chapron $M$, Ivanov $N$, Boissard $P$, Valéry $P$ (1992b) 3D reconstruction of plants. IAPR Workshop on Machine Vision Applications, December 7-9, Tokyo, Japan

Chapron M, Ivanov N, Boissard P, Valéry P (1993) Visualization of maize acquired from stereovision. IEEE SMC'93, October 17-20, Le Touquet, France

Faugeras O (1988) Quelques pas vers la vision artificielle en trois dimensions. Technique et science informatiques, AFCET, Bordas, 7, 547590

Goel N, Rozehnal I, Thompson R (1991) Vegetation canopies and arbitrary shapes: Computer generation and bidirectional reflectance calculations. Proc 5th Int Coloq Phys Measurements and Signatures in Remote Sensing, Courchevel (France), January 14-18, ESA SP-319, 409413

Greville T (1960) Some applications of the pseudo-inverse of matrix. SIAM Revue IIp, 1520

Ivanov N (1991) Application de la stéréovision à un couvert végétal en vue d'estimer des paramètres de structure. Mémoire de DEA, Université Paris 7, France, $62 \mathrm{p}$

Jia J, Krutz G (1992) Location of the maize plant with machine vision. J Agric Eng Res 52, 169181

Lewis P, Muller J P (1990) Botanical plant modelling for remote sensing simulation studies. Proc IGARSS' 90, Washington DC, USA, 21-24 May, vol 3, 1739-1742

Liu N (1991) Vision stéréoscopique : Calibration et mise en correspondance de primitives dans un univers polyedrique. Thèse de docteur ès sciences, Université de Paris Sud, France, $174 \mathrm{p}$

de Masson d'Autume G (1984) Corrélation numérique d'images quelconques selon les lignes quasi-épipolaires, par approximations successives. Bull Soc F Photogrammétrie Télédétection $95,23-32$ 
Moulia B, Sinoquet H (1993) Three-dimensional digitizing systems for plant canopy geometrical structure: a review. In: Crop Structure and Light Microclimate C Varlet-Grancher, R Bonhomme, $H$ Sinoquet ed) INRA éditions, 183-193

Porter JR (1984) A model of canopy development in winter wheat. J Agric Sci Camb 103, 383-392

Prevot L, Aries F, Monestiez P (1991) Modélisation de la structure géométrique du maïs. agronomie 11, 491-503

Prevot L, Brunet $Y$ (1993) Estimating the vertical profile of the leaf inclination distribution function: the silhouette method. In: Crop structure and Light Microclimate C Varlet-Grancher, R Bonhomme, $\mathrm{H}$ Sinoquet ed) INRA éditions, 195-200

de Reffye $P$, Edelin C, Françon J, Jaeger $M$, Puech C (1988) Plant models faithful to botanical structure and development. Comput Graphics 22, 151-158

Sinoquet H, Moulia B, Bonhomme R (1991) Estimating the 3-D geometry of a maize crop as an input of radiation models: comparison be- tween 3 dimensional digitizing and plant profiles. Agric Forest Meteorol, 55, 233-249

Sinoquet $\mathrm{H}$, Valmorin $M$, Cabo $X$, Bonhomme $R$ (1993) DALI: an automated laser distance meter system for measuring profiles of vegetation. Agric For Meteorol, 67, 43-64

Toscani G (1988) Système de calibration et parception du mouvement en vision artificielle. Thèse de docteur es sciences, l'Université de Paris Sud, France, 144p

Verhoef W (1984) Light scattering by leaf layers with application to canopy reflectance modeling: the SAIL model. Remote Sens Environ 16, 125-141

Welles JM, Norman JM (1991) An instrument for indirect measurement of canopy architecture. Agron $J$ 83, 818-825

Whisler JR, Acock B, Baker DN, Fye RE, Hodges $H F$, Lambert JR, Lemmon HE, McKinion JM, Reddy VR (1986) Crop simulation models in agronomic systems. Adv Agron 40, 141-208 